\title{
Proprietary Data Format
}

National Cancer Institute

\section{Source}

National Cancer Institute. Proprietary Data Format. NCI Thesaurus. Code C85871.

A file format that is controlled by a private person or org anization where the mode of presentation of its data is opaque and its specification is not publicly available. 